\title{
Adsorption of Catechol and Comparative Solutes on Hydroxyapatite
}

\author{
William M. Chirdon, ${ }^{1}$ William J. O’Brien, ${ }^{2}$ Richard E. Robertson ${ }^{3}$ \\ ${ }^{1}$ Macromolecular Science and Engineering and Department of Biologic and Materials Science, University of Michigan, \\ Ann Arbor, Michigan \\ 2 Department of Biologic and Materials Science, University of Michigan, 1011 North University Avenue, \\ Ann Arbor, Michigan 47109 \\ ${ }^{3}$ Department of Materials Science and Engineering and Macromolecular Science and Engineering Center, University of \\ Michigan, Ann Arbor, Michigan
}

Received 12 June 2002; revised 14 November 2002; accepted 4 December 2002

\begin{abstract}
Contemporary medical and dental adhesives often have difficulty sticking to wet surfaces or weaken with long-term exposure to water. Substantial research has been dedicated to finding a means of achieving adhesion in an aqueous environment. A study evaluates the adsorption of catechol relative to other chemical groups as means of gauging how effective they may be as adsorptive groups in adhesives. Contact angle and surface-tension measurements of solutions of catechols and other chemical groups were used to determine their works of adhesion. Adsorption isotherms were also constructed to ascertain Langmuir constants. Solutes containing catechol groups were compared to solutes containing other polar groups to see how well catechol adsorbs to hydroxyapatite, the mineral component of bones and teeth, relative to other chemical groups found in adhesives. The results of this study show that catechol and molecules containing catechol groups have higher rates and energies of adsorption to hydroxyapatite than do groups such as alcohols, amines, and carboxylic acids. ( 2003 Wiley Periodicals, Inc. J Biomed Mater Res Part B: Appl Biomater 66B: 532-538, 2003
\end{abstract}

Keywords: adsorption; adhesion; hydroxyapatite; catechol; surface chemistry

\section{INTRODUCTION}

Hydroxyapatite (HA) has been the subject of various studies for some time and continues to be targeted by biomaterials research. The adsorption of ions, proteins, and various complex biochemicals on HA has been thoroughly studied for biomaterial applications in tissue engineering and prosthetics. Adsorption and wetting phenomena are also important in adhesive technology, and hence, adsorption onto HA is relevant to the field of medical and dental adhesives. ${ }^{1}$ There have been extensive adsorption studies of various proteins and other macromolecules on HA, and the adsorption of simple chemical groups has been studied on various materials. However, the adsorption of simple chemical groups on HA, which has received less attention, is critical to under-

Correspondence to: William J. O'Brien, Department of Biologic and Materials Science, University of Michigan, 1011 North University Avenue, Ann Arbor, MI 47109 (e-mail: wjobrien@umich.edu)

Contract grant sponsor: NIDCR, National Institutes of Health, contract grant number: T32-DE07057

(C) 2003 Wiley Periodicals, Inc. standing how large molecules that contain these groups adsorb on HA.

This line of research has spawned from natural adhesive studies involving mussels. ${ }^{2,3}$ Mussels are capable of forming strong adhesive bonds to substrates in the presence of water. This scenario is analogous to that of the medical/dental adhesive problem, and is therefore the object of this investigation. From biological assays of the adhesive proteins, it has been discovered that the adhesive proteins have a relatively high occurrence of L-dopamine, which is an amino acid characterized by its catechol (3,4-dihydroxyphenyl) group. Catechol groups may serve a dual function in natural adhesives as cross-linking agents and as adsorptive groups. Previous research has shown catechol groups to adsorb strongly to many different materials through various energetic interactions, which can involve the hydroxyl groups and/or the aromatic rings. ${ }^{3}$ This study compares catechol and catecholcontaining chemicals to various other simple chemical groups such as amine, alcohol, and phenol. If catechol adsorption to HA proves greater than that of chemical groups currently used in adhesives, then this would support the theory that the catechol groups are an important part of the mussel adhesion 
mechanism. Furthermore, it would provide impetus for the development of an adhesive that incorporates catechol groups for improved adhesion and resistance to water.

Catechol groups have a strong affinity to hydrophilic polymers and a tremendous capacity for hydrogen bonding. ${ }^{4}$ Tannins are classic examples of this interaction, which displace water on the collagen backbone of leather in the tanning process. ${ }^{5}$ As metal-liganding agents, catechol groups have astronomically high cumulative stability constants $(\mathrm{p} K)$ for many ligands such as $\mathrm{Al}^{3+}(\mathrm{p} K=47)$ and $\mathrm{Fe}^{3+}(\mathrm{p} K=45)$ at $20{ }^{\circ} \mathrm{C}$ and $\mathrm{pH} 10 .{ }^{3}$ Such is the affinity of catechol for these metals, that it should have the ability to displace cyanide and oxide from the $\mathrm{Fe}^{3+}$ cation. ${ }^{3}$ Considering the affinity with which catechol groups bind to these various hydrophilic surfaces and molecules, their potential as adsorptive groups for HA certainly merits investigation.

\section{MATERIALS AND METHODS}

\section{Chemicals}

For the contact-angle study, ceramic HA from American International Chemical with a reported 20-micron particle size was used. The HA source for the adsorption experiments was tribasic calcium phosphate. (Aldrich Chemicals, Inc.) Most of the adsorption experiments used HA with a specific surface area of $83.25 \mathrm{~m}^{2} / \mathrm{g}$. HA purchased later had a specific surface area of $75.82 \mathrm{~m}^{2} / \mathrm{g}$ and was used for the study of hexanoic acid. The average specific surface areas were quantified by BET. Magnesium oxide was purchased from Mallinckrodt Chemical Works. Dimethyl sulfoxide was purchased from Fisher. Absolute ethanol (200 proof) was obtained from Pharmco Products, Inc. All other chemicals were purchased from Aldrich Chemicals, Inc.

The solutes for this comparison study are phenol (hydroxybenzene), catechol (1,2-dihydroxybenzene), pyrogallol (1,2,3-trihydroxybenzene), 3-hydroxytyramine, gallotannic acid (contains pyrogallol group), hexanoic acid, hexylamine, and hexanol. Phenol, catechol, and pyrogallol are expected to show the effects of the first, second, and third aromatic hydroxyl groups on adsorption, respectively. 3-Hydroxytyramine is a catechol with an amine group. Gallotannic acid is an example of a large, complex molecule that contains catechol, and shows some of the potential effects of incorporating catechols into large molecules. Hexanoic acid, hexylamine, and hexanol represent small molecules containing carboxylic acid, amine, and alcohol groups, respectively. The six-carbon length was chosen to have a size and mass comparable to catechol.

\section{Contact Angle and Surface Tension}

Via Young's equation, the contact angle and surface tension are used to calculate the work of adhesion $\left(W_{\mathrm{S} / \mathrm{L}}\right)$ for all of the selected chemical groups in concentrated solutions. The theory is physically meaningful and employs a force balance of surface tensions, Eq. (1), and the Dupré relation, Eq. (2), to achieve Young's equation, Eq. (3). ${ }^{6}$

$$
\begin{gathered}
\gamma_{\mathrm{S} / \mathrm{A}}=\gamma_{\mathrm{S} / \mathrm{L}}+\gamma_{\mathrm{L} / \mathrm{A}} \cos \theta, \\
W_{\mathrm{S} / \mathrm{L}}=\gamma_{\mathrm{S} / \mathrm{A}}+\gamma_{\mathrm{L} / \mathrm{A}}-\gamma_{\mathrm{S} / \mathrm{L}}, \\
W_{\mathrm{S} / \mathrm{L}}=\gamma_{\mathrm{L} / \mathrm{A}}(1+\cos \theta),
\end{gathered}
$$

where $W_{\mathrm{S} / \mathrm{L}}=$ work of adhesion, $\gamma=$ surface tension, and $\theta=$ contact angle. Subscripts denote interface: $S=$ solid, $L=$ liquid, and $A=$ air.

The sample preparation for this experiment starts by using a mortar and pestle to mix $0.27 \mathrm{~g}$ of $\mathrm{HA}$ and $0.03 \mathrm{~g} \mathrm{MgO}$. This mixture was then pressed by hand in a $25 \times 6-\mathrm{mm}(1 \times$ 0.25 in.) mold and sintered at $1121{ }^{\circ} \mathrm{C}$ for $20 \mathrm{~min}$ in air. The $\mathrm{MgO}$ was needed to decrease brittleness and porosity. These sintering conditions are below the temperature range where HA undergoes dehydroxylation. ${ }^{7}$

The angles of contact of a HA slab dipped into solutions with concentrations of $0.1,0.2$, and $0.3 \mathrm{~g} / \mathrm{ml}$ of the solutes were measured at room temperature. The surface tension was measured via the Wilhelmy plate method with a Rosano surface tensiometer manufactured by the Biolar Corporation. Dimethyl sulfoxide (DMSO) was chosen as the solvent for all of the contact angle measurements because it was the only nonvolatile solvent that had an appreciable contact angle with HA and that would dissolve all of the solutes chosen without reacting with them. Twenty-four contact-angle measurements were taken for each solution.

\section{Adsorption Isotherms}

These experiments consisted of adsorption experiments in dilute solutions where the adsorption isotherms were calculated from mass balances of initial and final solutions. The adsorption isotherms were then fitted by the Langmuir equation to get two constants: $K$ and $N_{o}$. $K$ represents the ratio of the rate constants of adsorption to desorption, and $N_{o}$ represents the amount in the fully adsorbed monolayer. The Langmuir equation is also simple and meaningful, but it does involve assumptions that may or may not prove to be true. The quality of the fits to the Langmuir equation will be discussed as a part of the results. The Langmuir equation first assumes simple first-order rates of adsorption and desorption shown in Eqs. (4) and (5), respectively:

$$
\begin{gathered}
r_{a}=k_{a} x(1-\theta), \\
r_{d}=k_{d} \theta .
\end{gathered}
$$

The Langmuir equation then assumes a steady state,

$$
r_{a}=r_{d}, \quad k_{a} x(1-\theta)=k_{d} \theta,
$$

which can be rearranged to 


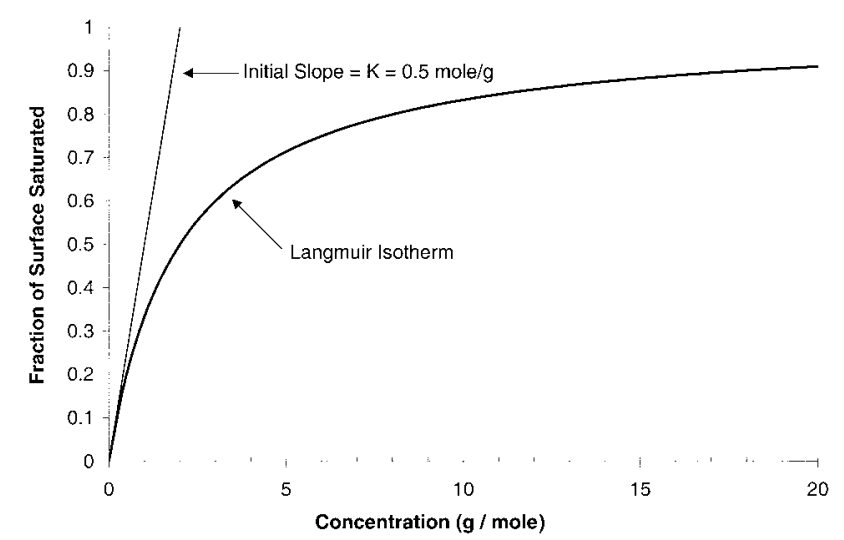

Figure 1. Example of a Langmuir isotherm. Note that the slope approaches $\mathrm{K}$ as the concentration approaches zero.

$$
\theta=\frac{\left(k_{a} / k_{d}\right) x}{1+\left(k_{a} / k_{d}\right) x}
$$

Then, with $K=k_{a} / k_{d}$,

$$
\theta=\frac{K x}{1+K x}
$$

Here

$$
\begin{aligned}
& r=\text { rate } \\
& k=\text { first-order rate constant } \\
& x=\text { concentration of adsorbate } \\
& N=\text { amount of surface sites occupied } \\
& N_{o}=\text { total amount of surface sites } \\
& \theta=\text { fraction of layer occupied, }\left(\theta=N / N_{o}\right)
\end{aligned}
$$

Subscripts a and d denote adsorption and desorption, respectively.

$K$ can be interpreted in several ways. It is defined as the ratio of the adsorption to desorption rate constants and can be thought of as an affinity constant. $K$ represents how quickly the layer saturates. (When $K x=1$, the layer is $50 \%$ saturated, so $K=1 / x_{\theta}=50 \%$.) In addition, $K$ is equal to the initial slope of the Langmuir equation. ( $d \theta / d x=K$ as $x$ approaches zero.)
So, if the Langmuir equation fits the data, then these highly relevant constants can be calculated. A graphical representation of this equation is shown in Figure 1.

The objective of this experiment is to determine the adsorption isotherms for the various solutes on HA. Water could not be used due to the reactivity with some of the solutes. Ethanol was chosen as the solvent for these experiments because it could dissolve at least $0.001 \mathrm{~g} / \mathrm{ml}$ of each solute. Ethanol is also more similar to the aqueous systems found in vivo, but it does not readily react with the solutes. Hexanol was not used in this experiment since the adsorption of alcohol groups is compared to others implicitly by using ethanol as a solvent.

Ten samples with $0.5 \mathrm{~g}$ of HA and $10 \mathrm{ml}$ of solution in $20-\mathrm{ml}$ vials were run at a time. The samples were tumbled end over end at a rate of $30 \mathrm{rev} / \mathrm{min}$ at room temperature. After tumbling for $1 \mathrm{~h}$, the contents of the vials were then poured into test tubes and centrifuged at $4000 \mathrm{rpm}$ for $45 \mathrm{~min}$ to settle out the HA. The clear solution was then removed via pipette for an appropriate analysis that depended on the solute. The concentrations of the aromatic solutes were determined by UV analysis using a UV160U UV-visible recording spectrophotometer made by Shimadzu. Hexylamine concentrations were found through $\mathrm{pH}$ determination with Fisher's Universal Indicator Solution and a Macbeth ColorEye 7000 spectrophotometer. Hexanoic acid concentrations were found by using a $\mathrm{pH}$ meter. By taking the difference between the final and initial concentrations and multiplying by the volume, the amount adsorbed from each solution was calculated. The mass of HA is known, and the specific surface area was determined by BET. Hence, the adsorption of solute per unit of area is known as a function of equilibrium (final) concentration. $K$ and $N_{o}$ can then be determined by the slope and intercept from a plot of inverse adsorption as a function of inverse concentration.

Example adsorption calculations for a set of catechol data are demonstrated in Table I. Using prepared catechol solutions of known concentration, the height of the UV absorp-

\begin{tabular}{|c|c|c|c|c|c|c|c|c|}
\hline $\begin{array}{c}\text { Conc(init) } \\
(\mathrm{g} / \mathrm{l})\end{array}$ & $\begin{array}{c}\text { 279-nm } \\
\text { Absorbance }\end{array}$ & $\begin{array}{c}\text { Conc(meas) } \\
(\mathrm{g} / \mathrm{l})\end{array}$ & Dilution & $\begin{array}{c}\text { Conc }(E q) \\
(\mathrm{g} / \mathrm{l})\end{array}$ & $\begin{array}{l}\mathrm{HA} \\
(\mathrm{g})\end{array}$ & $\begin{array}{l}\text { Adsorbed } \\
(\mathrm{g})\end{array}$ & $\begin{array}{c}\text { Adsorbed/HA } \\
(\mathrm{g} / \mathrm{g})\end{array}$ & $\begin{array}{l}\text { Adsorbed/Area } \\
\left(\mathrm{g} / \mathrm{m}^{2}\right) 10^{6}\end{array}$ \\
\hline 1 & 1.2276 & 0.055705 & 15 & 0.835578 & 0.429 & 0.001644 & 0.003833 & 46.04 \\
\hline 0.2 & 1.2329 & 0.055943 & 1 & 0.055943 & 0.485 & 0.001441 & 0.002970 & 35.68 \\
\hline 0.1 & 0.1438 & 0.006525 & 1 & 0.006525 & 0.53 & 0.000935 & 0.001764 & 21.19 \\
\hline 0.05 & 0.0264 & 0.001200 & 1 & 0.001200 & 0.469 & 0.000488 & 0.001041 & 12.50 \\
\hline
\end{tabular}
tion peak at $279 \mathrm{~nm}$ is thereby known as a function of catechol concentration. This calibration curve is shown as Figure 2. With the calibration curve, the concentration of the decanted adsorption solution can be calculated. Dilution was necessary for the more concentrated solutions to stay within the range of the UV detector. The equilibrium concentration

TABLE I. Calculation of Catechol Adsorption Isotherm 


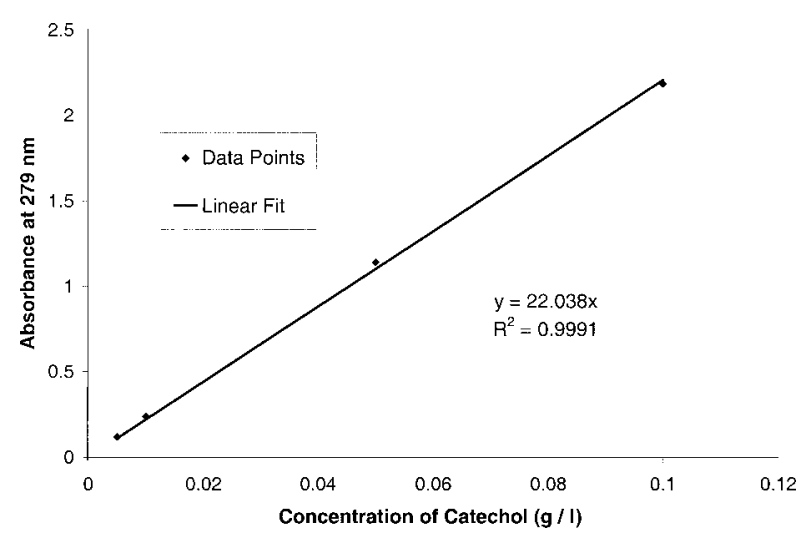

Figure 2. Calibration curve for the determination of catechol concentration. The absorbance at $279 \mathrm{~nm}$ directly relates to catechol concentration.

can then be calculated the data. The difference between the initial and equilibrium concentrations multiplied by the solution volume yields the mass adsorbed. The mass adsorbed per area is known from the known mass of HA and its measured BET area. Thus, the mass adsorbed per unit of HA area is a known function of equilibrium concentration, as shown in Figure 3. This isotherm can then be fitted to the Langmuir equation via regression of inverse adsorption as a function of inverse concentration. The Langmuir equation regression curve is also plotted in Figure 3.

\section{RESULTS}

\section{Contact Angle}

Summaries of the contact angle and surface-tension data are displayed in Figure 4. From Young's equation [Eq. (3)], the work of adhesion can be calculated. The sign convention used is a positive number for exothermic, strong adsorption. A summary of the average works of adhesion data with corresponding standard deviations is shown in Table II for the three concentrations.

Due to complications of the effects of concentration, the comparison was made at the highest concentration attainable, $0.3 \mathrm{~g} / \mathrm{ml}$. In Table II, the works of adhesion for the concentrated solutions along with the work of adhesion of DMSO are displayed. One should note that the four solutes containing catechol or pyrogallol groups have the highest $K$ values.

\section{Adsorption Isotherms}

The summary of the Langmuir constants and the Pearson product moment correlation coefficient $R$ from regression for each solute is listed in Table III. $R$ was calculated as

$$
R=1-\frac{n\left(\sum X Y\right)-\left(\sum X\right)\left(\sum Y\right)}{\sqrt{\left[n \sum X^{2}-\left(\sum X\right)^{2}\right]\left[n \sum Y^{2}-\left(\sum Y\right)^{2}\right]}},
$$

where $X$ and $Y$ are the ordinate and coordinate for a data set of $n$ points.

The Langmuir equation does not presume units of $N_{o}$ and $K$, so the results are tabulated with the constants in molar units, which are more appropriate for most analyses than mass units. Table III lists the solutes with their regression results and is sorted by $K$. Plots of some of the Langmuir isotherms using the coefficients from regression are shown in Figure 5.

Various points of interest should be noted when examining these figures. Judging by the $R$ values, the Langmuir equation has proven to fit the data generally well, as shown quantitatively in Table III. Phenol is not modeled well as a Langmuir isotherm. The equation fits the data well statistically, but it does so with a large $N_{o}$ and miniscule $K$, as the data is essentially linear over the concentration range tested. Phenol is probably not adsorbing on HA, and the miniscule amount that disappeared may be a result of precipitation or some other removal not related to the adsorption on the HA surface.

\section{DISCUSSION}

The aim of this investigation was to efficiently survey various chemical groups to determine how well they adsorb to HA relative to each other. The solutes cannot be simply sorted by which adsorbs best on HA, because this depends on the specific adsorptive property of comparison, the solvent, concentration, and other experimental conditions. This study provides evidence that catechol groups adsorb more readily than other representative groups on HA in terms of both energy and kinetics. For the chemical design of an adhesive, it is far more important for an adsorptive group to adsorb more readily and stronger (large $K$ and $W_{\mathrm{S} / \mathrm{L}}$ ) than to adsorb a greater amount (large $N_{o}$ ). The amount adsorbed is more strongly dependent on molecular mass, the types of adsorption sites, and whether molar or mass amounts are being compared. Therefore, one should note that catechols do not

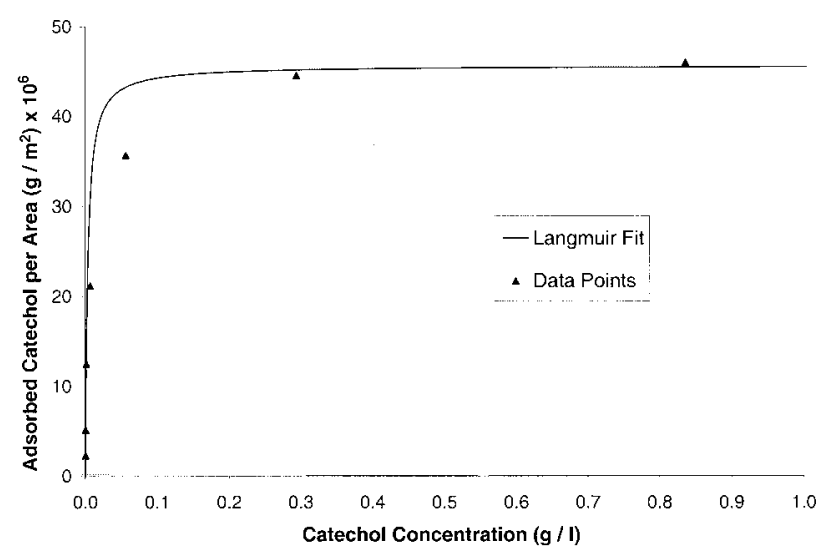

Figure 3. Adsorption isotherm for catechol on HA. The Langmuir model is fitted to the resultant data. 


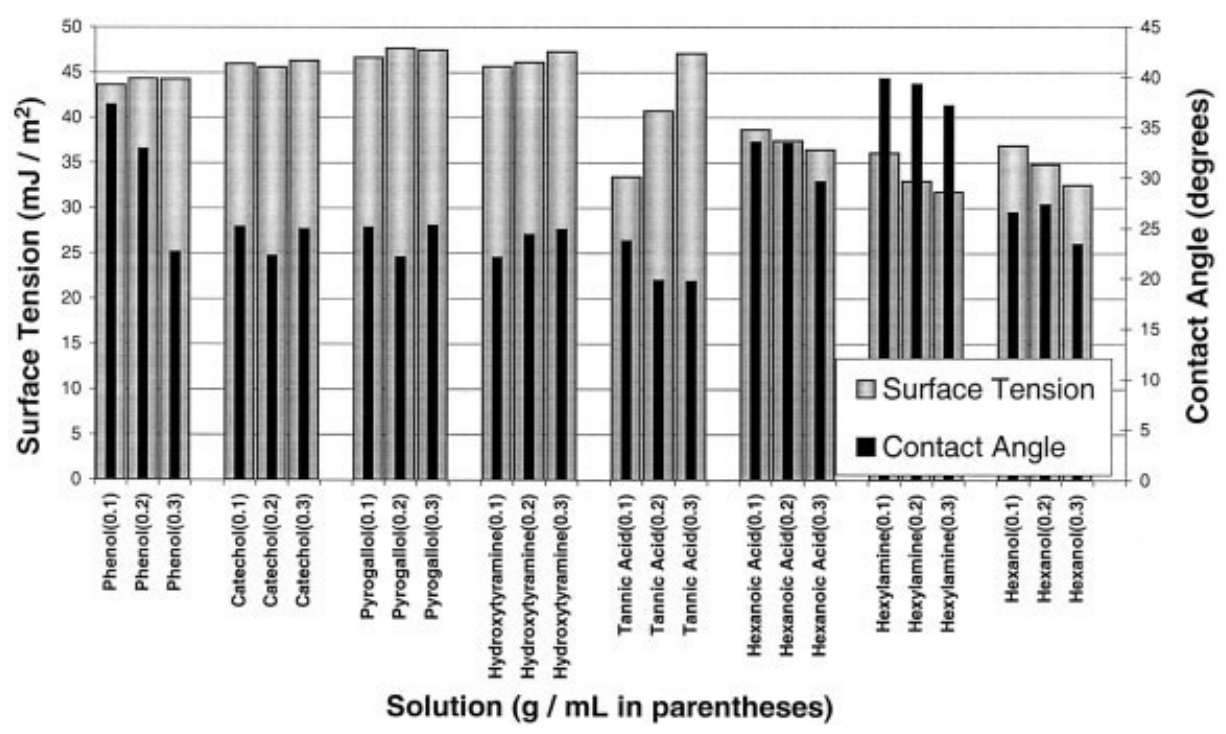

Figure 4. Surface-tension and contact-angle data used for the determination of work of adhesion.

necessarily adsorb more in terms of the amount required for saturation, but they adsorb more readily and approach saturation at lower concentrations.

\section{Contact Angle}

One concern regarding these results is that if the work of adhesion is linearly extrapolated back to zero concentration, the result is not that of the work of adhesion for the solvent. The cause of this oddity is not known, but the following is a possible explanation. From the adsorption results, these concentrations are found to be orders of magnitude above that required to saturate the HA surface. Originally, the work was hypothesized to be a linear function of concentration with the zero intercept equaling the solvent work of adhesion. If this were the case, the excess work per mole of solute could be calculated. However, none of the solutions displayed a direct relation of excess work with concentration. Rather, some displayed a seemingly linear effect without having zero intercepts of the solvent work of adhesion, while others were constant (independent of concentration). The solutions that were relatively constant with respect to concentration probably had similar energies of adhesion at various large concentrations because the interfaces are identically saturated. Hence, the nonzero intercept of excess work as a function of concentration is the energy from forming the saturated layer. The slope of this function may be due to secondary effects of concentration on the work of adhesion, such as the energies of formation of multiple layers on top of a monolayer.

This hypothesis can be extended to explain why the solutes that did not adsorb strongly tended to have larger slopes with respect to concentration, and those that adsorbed strongly were nearly constant with respect to concentration. The solutions with high $K$ values may all have the same adsorbed monolayer, and hence, the same work of adhesion, whereas those with low $K$ values were not entirely adsorbed and are still undergoing adsorption at this concentration. Although these results may provide some insight to the structure of the adsorbed layers, it would be imprudent to try to deduce such complex molecular phenomena solely from a single macroscopic experiment.

TABLE II. Works of Adhesion $\left(\mathrm{mJ} / \mathrm{m}^{2}\right)$

\begin{tabular}{|c|c|c|c|c|c|c|}
\hline & \multicolumn{2}{|c|}{$0.1 \mathrm{~g} / \mathrm{ml}$} & \multicolumn{2}{|c|}{$0.2 \mathrm{~g} / \mathrm{mL}$} & \multicolumn{2}{|c|}{$0.3 \mathrm{~g} / \mathrm{mL}$} \\
\hline & Mean & $\mathrm{sd}(+/-)$ & Mean & $\mathrm{sd}(+/-)$ & Mean & $\operatorname{sd}(+/-)$ \\
\hline Tannic acid & 64.06 & 2.33 & 79.09 & 3.36 & 91.33 & 3.20 \\
\hline Pyrogallol & 88.90 & 4.64 & 91.83 & 1.74 & 90.35 & 3.34 \\
\hline Hydroxytyramine & 87.92 & 1.85 & 88.10 & 3.00 & 90.05 & 3.79 \\
\hline Catechol & 87.54 & 4.98 & 87.79 & 3.65 & 88.23 & 2.93 \\
\hline Phenol & 66.38 & 4.18 & 81.56 & 3.15 & 85.08 & 4.29 \\
\hline DMSO & 82.72 & 5.81 & 82.72 & 5.81 & 82.72 & 5.81 \\
\hline Hexanoic acid & 70.86 & 4.10 & 68.61 & 8.11 & 68.19 & 5.95 \\
\hline Hexanol & 69.85 & 2.65 & 65.74 & 2.60 & 62.32 & 2.41 \\
\hline Hexylamine & 63.75 & 5.55 & 58.45 & 6.13 & 57.05 & 3.19 \\
\hline
\end{tabular}


TABLE III. Regressed Langmuir Constants (Sorted on the Basis of $K$ )

\begin{tabular}{lccc}
\hline & $\mathrm{K}(\mathrm{ml} / \mathrm{mol})$ & $\mathrm{N}_{\mathrm{o}}\left(\mathrm{mol} / \mathrm{m}^{2}\right)$ & $R$ \\
\hline Tannic acid & $1.86 \mathrm{E}+09$ & $3.58 \mathrm{E}-07$ & 0.995 \\
Catechol & $3.31 \mathrm{E}+07$ & $4.39 \mathrm{E}-07$ & 0.989 \\
Hydroxytyramine & $2.23 \mathrm{E}+07$ & $4.97 \mathrm{E}-07$ & 0.983 \\
Hexanoic acid & $1.73 \mathrm{E}+07$ & $1.56 \mathrm{E}-06$ & 0.997 \\
Pyrogallol & $4.08 \mathrm{E}+06$ & $1.13 \mathrm{E}-06$ & 0.975 \\
Hexylamine & $3.83 \mathrm{E}+06$ & $4.56 \mathrm{E}-07$ & 0.996 \\
Phenol & $1.24 \mathrm{E}+04$ & $5.10 \mathrm{E}-06$ & 0.989 \\
\hline
\end{tabular}

\section{Adsorption Isotherms}

As seen by the Pearson product moment correlation coefficients of the fits, Eq. (11), and by visual inspection of the graphs, the Langmuir equation describes the data well. One way in which the data does not fit the equation well, however, is that most plots have a positive, constant slope at high concentrations. Most likely, this is due to such phenomena as precipitation, micelle formation, or some other depletion of concentration unrelated to adsorption on HA. The Langmuir equation is intended for use with dilute solutions, so it is not surprising that it does not model the linear behavior at higher concentrations. The primary objective of this experiment is the comparison of the relative rates of adsorption to desorption of the solutes. Numerically, this is judged from the values of $K$, which are determined from the low regions of the isotherms. Therefore, the slight slopes of the high concentration data are not of great concern.

For purposes of comparison, one must be aware of the distinction between the comparisons based on mass versus molar units. Direct mathematical manipulation results in mass units, but the more relevant comparison is in molar units. Again, the three groups that adsorbed the most readily, judging by $K(\mathrm{ml} / \mathrm{mole})$, all contained catechol or pyrogallol groups. Catechol was the most readily adsorbed simple molecule of low molecular weight. Tannic acid is a large, complex molecule, which adsorbs the most readily on both a molar and mass scale. Tannic acid was suspected to adsorb readily, as it is a component in coffee and tea that stains teeth.

The work of adhesion and the adsorption isotherm studies are related in their objective of comparing the adsorption of solutes, but at this point, it is prudent to emphasize their differences to better understand the results. A critical difference is the use of different solvents, which affects the nature of the solute, surface, and adsorption sites. Properties such as polarity and $\mathrm{pH}$ can strongly affect the protonation of solutes and affect the mechanisms, rates, and amounts of adsorption. One should anticipate that the two experiments would have slightly different results, considering that they were designed to determine different physical properties in different solvents. The contact-angle study is used to estimate the relative energies of adsorption, while the adsorption isotherms are used to estimate the relative rates of adsorption/desorption and the amount adsorbed. Despite these differences, both studies show catechol/pyrogallol groups adsorb better than other groups such as amines, alcohols, and carboxylic acids, based on their works of adhesion and relative rates of adsorption/desorbtion for both solvents and both analytical methods.

Catechol, 3-hydroxytyramine, and pyrogallol have similar works of adhesion, which seems to suggest pyrogallol's third hydroxyl group and 3-hydroxytyramine's amine group are vestigial to a first approximation. This is relevant to determining the mechanism, in that a third hydroxyl group or additional amine group has little effect on the adsorption, which appears to be based on the attachment of two hydroxyl groups. The adsorption of phenol, however, was far below that of catechol and pyrogallol, which further supports divalent mechanisms. The adsorption rate, energy, and amount (in mass units) of tannic acid was substantially greater than that of the smaller molecules. This shows that catechols can adsorb even better when incorporated into large molecules.

Mechanistically, intramolecular cooperativity of the same catechols on the same molecule may be part of the explanation. In addition, large molecules have the advantage of having less of an entropic barrier to adsorption. Complex mechanisms of adsorption may also have been tailored by nature for this biological molecule. Carboxylic acid, which is often used as an adsorption group for HA in dental adhesives, generally adsorbed well but not as well as the catecholcontaining groups.

To develop further insight into the nature of catechol adsorption to hydroxyapatite, the apparent area occupied per molecule was calculated. This is a straightforward calculation from the primary results of the experiment. When $N_{o}$ is calculated in units of moles of adsorbate per area, multiplying by Avogadro's number and then taking the inverse yields the apparent area occupied per molecule. The apparent occupied area per molecule for catechol was $3.8 \mathrm{~nm}^{2}$, which is far greater than a theoretical area per molecule, which is approximately $0.067 \mathrm{~nm}^{2}$, based on theoretical bond lengths and atomic radii. Because the theoretical value is about $2 \%$ of the experimental value, the logical conclusion is that only $2 \%$ of

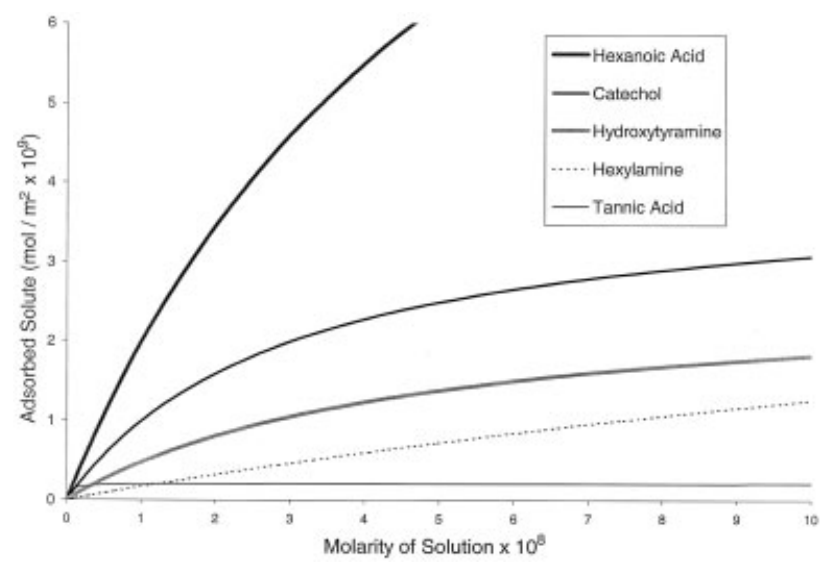

Figure 5. Fitted Langmuir models for the solutes. For clarity, the key has been sorted by the adsorption at the highest concentration shown. 
the surface area determined by BET is available to catechol adsorption. In an analogous experiment involving catechol adsorption to alumina, McBride and Wesselink have deduced that catechol primarily adsorbs to the edges and not the faces of alumina. ${ }^{8}$ These results suggest similar adsorption behavior for catechols on HA.

Researchers have proposed various mechanisms as explanations for the excellent adsorption of catechol groups on HA. However, the fact that catechol readily and competitively adsorbs to various hydrophilic and metallic surfaces is not in dispute. Misra maintains that the adsorption can be explained via hydrogen bonding. ${ }^{9}$ Although hydrogen bonding may explain this effect, the potential electrostatic interactions of de-protonated catechol groups and the effects of the aromatic rings should not be neglected.

Another possible mechanism that has not been emphasized in the literature may also be in effect. The total energy of adsorption is not simply the change in energy from solute molecule in solution to a solute molecule on a substrate. Rather, an often-neglected aspect of adsorption energy is the cooperative energy of the solute molecules interacting with other solute molecules adsorbed on the surface. Depending on the presence and position of the hydrogen atoms on the hydroxyl groups on catechol, it is a planar or near planar molecular group. This would allow the groups to stack closely, side by side, and benefit from substantial energies of interaction between the aromatic rings of the catechol groups. This cooperativity allows adsorbed groups to pull other catechol groups from solution into the monolayer and to be retained. This mechanism, which can be combined with metal-ligand, electrostatic, or hydrogen bonding mechanisms involving the surface, can explain the heightened rate and energy of adsorption.

Although these experiments do not directly indicate the precise molecular mechanism for catechol adsorption on HA, some deductions can be made from the experimental data. These results suggest that catechol groups cooperatively bind via divalent hydrogen bonds along the HA crystal edges. Spectroscopic and calorimetric studies of the adsorption behavior would lead to improved understanding of the adsorption mechanism.

\section{CONCLUSIONS}

The results provide impetus for researching catechol for adhesive and adsorptive applications, as this work has shown that catechol groups have superior adsorptive properties relative to the other representative solutes in this experiment. Solutions containing catechols have displayed the highest works of adhesion, which indicates that adhesives containing catechols should similarly have excellent energies of adhesion and superior wetting properties on hydroxyapatite. Catechols also adsorbed more readily, as seen in the adsorption isotherms, which means that catechols are likely to successfully compete for adsorption sites. These results support the hypothesis that the catechol of the dopamine group found in marine adhesive proteins plays an important role in the adhesion mechanism. Therefore, the primary ramification of this study should be to inspire further research into how catechols may be incorporated into an effective adhesive system.

The authors would like to thank Professor Richard Laine for the use of his BET equipment and Mr. Thomas Hinklin for his instruction regarding its operation.

\section{REFERENCES}

1. Misra DN. Adsorption on and surface chemistry of hydroxyapatite. New York: Plenum; 1982.

2. Deacon MP, Davis SS, Waite JH, Harding SE. Structure and mucoadhesion of mussel glue protein in dilute solution. Biochemistry 1998;37:14108-14112.

3. Waite, JH. Nature's underwater adhesive specialist. Chemtech 1987;17:692-697.

4. Bienkiewicz, KJ. Physical chemistry of leather making. Malabar, FL: Kreiger; 1983. pp 385-406.

5. Hagerman AE, Butler LG. The specificity of proanthocyanidinprotein interactions. J Biol Chem 1981;256:4494-4497.

6. Davies JT, Rideal EK. Interfacial phenomena. New York: Academic; 1963.

7. Muralithran G, Ramesh S. The effects of sintering temperature on the properties of hydroxyapatite. Ceram Int 2000;26:221-230.

8. McBride MB, Wesselink LG. Chemisorption of catechol on gibbsite, boehmite, and noncrystalline alumina surfaces. Environ Sci Technol 1988;22:703-708.

9. Misra DN. Adsorption from solutions on synthetic hydroxyapatite: Nonaqueous vs. aqueous solvents. J Biomed Mater Res 1999;48:848-855. 